\title{
ENDOGENOUS ENDOPHTHALMITIS ASSOCIATED WITH INTRAVENOUS DRUG ABUSE
}

\section{Seven-Year Experience at a Tertiary Referral Center}

\author{
PAUL P. CONNELL, FRCSI (OPHTH), ${ }^{\dagger} \dagger$ EVELYN C. O'NEILL, MRCOPHTH, ${ }^{*} \dagger$ \\ F. M. AMIRUL ISLAM, PHD,* ROBERT BUTTERY, FRANZCO, $\dagger$ MARK MCCOMBE, FRANZCO, $\dagger$ \\ ROHAN H. ESSEX, FRANZCO, $\uparrow$ EDWARD ROUFAIL, FRANZCO, $\dagger$ STEPHEN LASH, FRCOPHTH, $\dagger$ \\ BERNARD WOLFFE, FCOPHTH SA,$\dagger$ BEN CLARK, FRANZCO,$\dagger$ DANIEL CHIU, FRANZCO, $\uparrow$ \\ WILLIAM CAMPBELL, FRANZCO,* PENELOPE ALLEN, FRANZCO $\dagger$
}

\begin{abstract}
Purpose: Intravenous drug use (IVDU) is a known risk factor for endogenous endophthalmitis. Endogenous fungal endophthalmitis (EFE) is emerging as a common problem among this community. We describe the management and visual outcomes of acute IVDU-associated EFE.

Methods: A prospective consecutive case series of 19 patients presenting with presumed acute IVDU-associated EFE from 2001 to 2007 to the Royal Victorian Eye and Ear Hospital was included. All data were collected in a standardized manner. Outcome measures included visual acuity, microbial profiles, and vitrectomy rate.

Results: Nineteen cases of IVDU-associated EFE were identified. Eight of these (42\%) were men, and the mean age was 32.7 years ( $S D \pm 8.0$ years). Presenting visual acuity ranged from $6 / 6$ to perception of light, with $58 \%$ having a visual acuity of $6 / 48$ or less at presentation. Thirteen (68.4\%) were culture positive with all cultures identifying Candida species, and $52.7 \%$ underwent vitrectomy. Fifty percent of subjects overall achieved a final visual acuity of $6 / 18$ or better. Men demonstrated improved visual acuity when compared with women $(P=0.04)$. Age had no effect on final acuity.

Conclusion: Intravenous drug use is a significant risk factor for developing EFE. Good visual outcomes can be achieved with early treatment, often with intravitreal therapy alone.

RETINA 30:1721-1725, 2010
\end{abstract}

E ndogenous endophthalmitis is an intraocular infection in which the causative organism reaches the eye through disseminated hematogenous spread. ${ }^{1,2}$ It is estimated that approximately 2 to $10 \%$ of all endophthalmitis cases are endogenous. The majority of cases are associated with an immunocompromised state, debilitating disease, invasive procedures, malignancy, and intravenous drug use (IVDU). ${ }^{3,4}$

\footnotetext{
From the *Centre for Eye Research Australia, University of Melom the Royal Victorian Eye and Ear Hospital, East Melbourne, Australia.

The authors have no conflicts of interest to disclose.

Reprint requests: Paul P. Connell, FRCSI(OPHTH), Vitreoretinal Unit, The Royal Victorian Eye and Ear Hospital, 32 Gisborne St, East Melbourne, Victoria, Australia 3002; e-mail: drpaulconnell@gmail.com
}

Endogenous fungal endophthalmitis (EFE) is a known complication of disseminated fungal infections and is potentially blinding. ${ }^{1,2}$ It is more prevalent than previously believed, especially in developing countries. ${ }^{5}$ Most reported cases of EFE occur in immunocompromised hosts. The frequency of EFE among patients with known systemic fungal infection has been reported to vary between 2 and $45 \% .^{3}$ The number of people living in a prolonged immunocompromised state is increasing. Advances in medical therapy and diagnosis have led to increased incidence of this condition. In clinical practice, many patients with EFE are misdiagnosed or diagnosed late with a resultant delay in treatment and poor visual prognosis. ${ }^{1}$ 


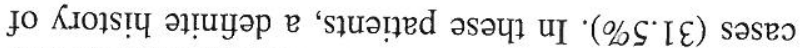

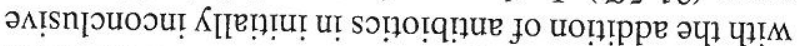

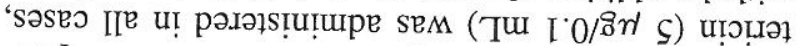

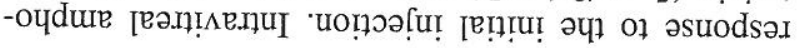

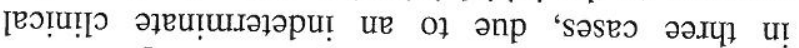

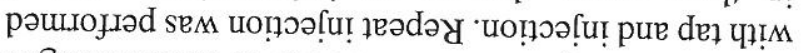

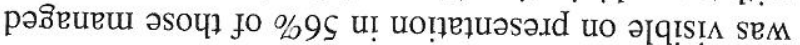

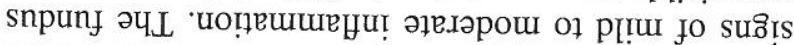

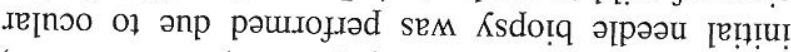

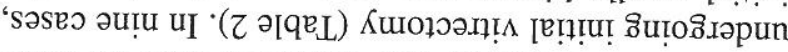

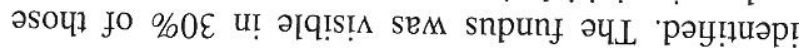

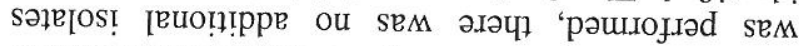

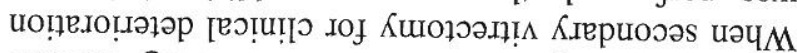

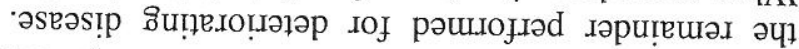

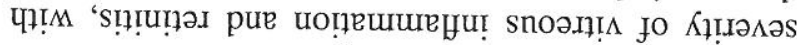

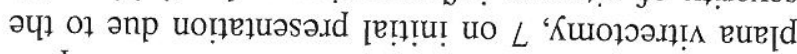

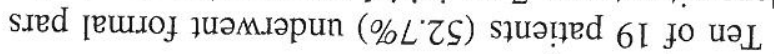

( ( ә ә

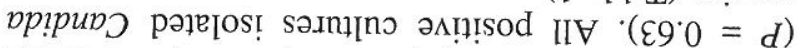

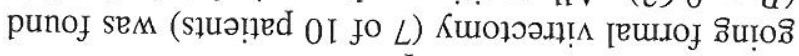

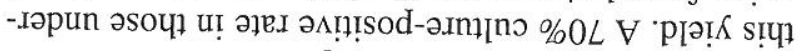

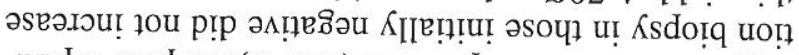

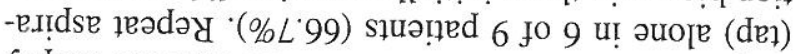

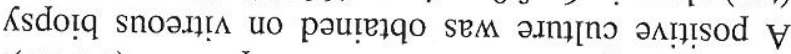

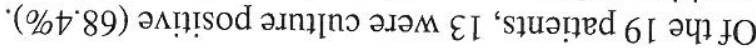

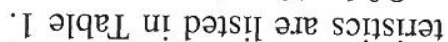

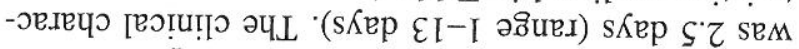

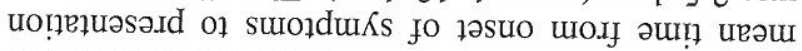

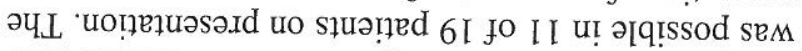

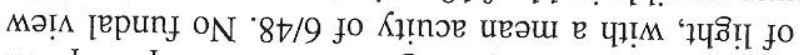

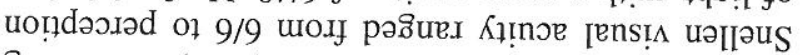

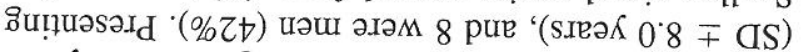

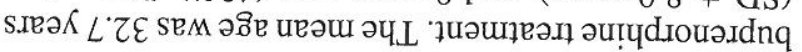

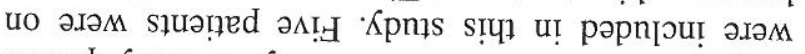

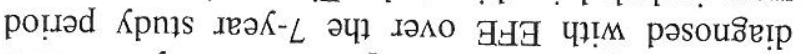

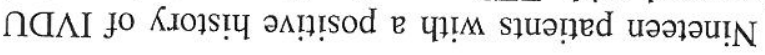

\section{Sł[nsวy}

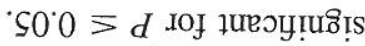

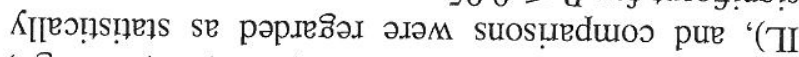

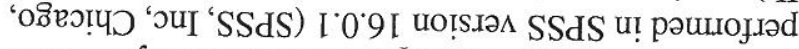
ว.әм Sәs Кㄹ

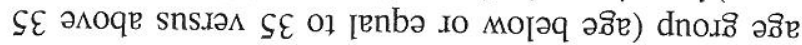

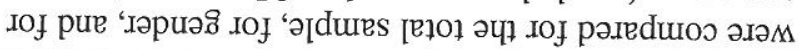
sə!̣!n

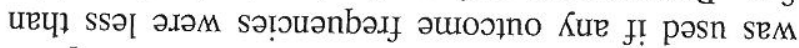

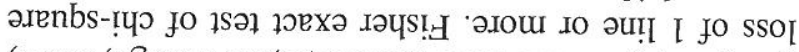

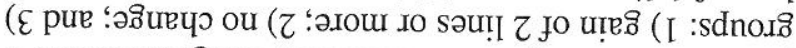

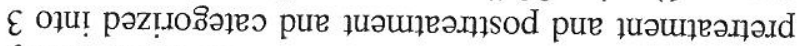

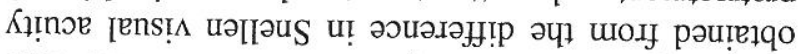

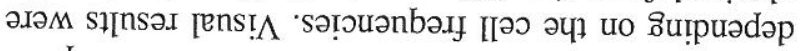

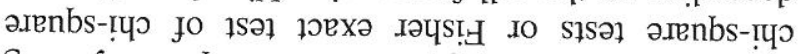
ô!

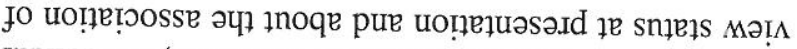

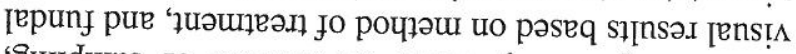

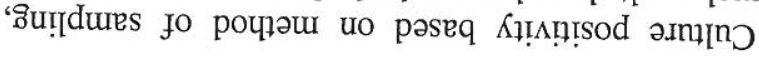

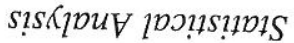

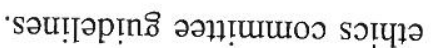

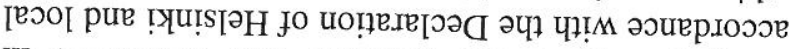

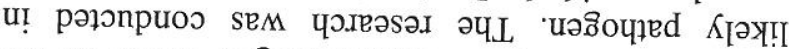

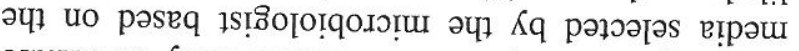

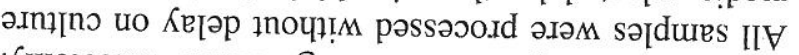

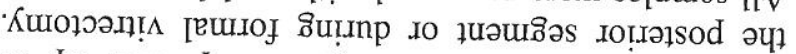

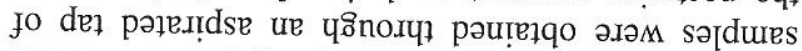

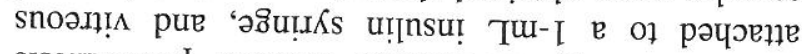

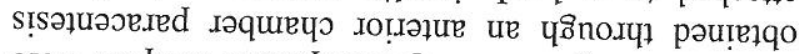

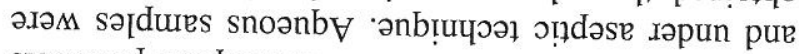

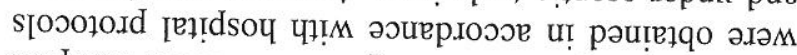

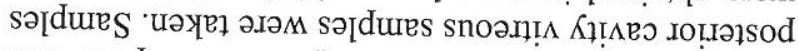

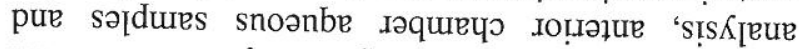
[втqолотฺ

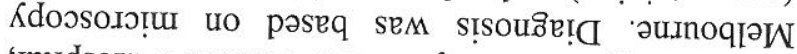

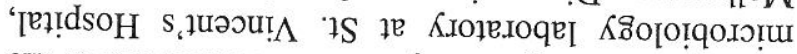

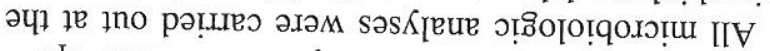

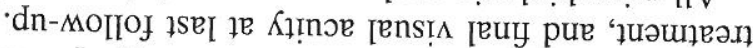

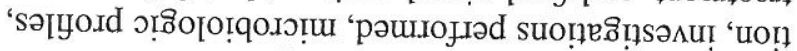

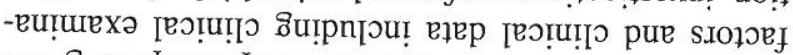

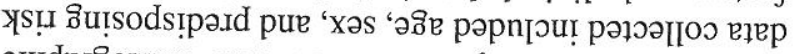

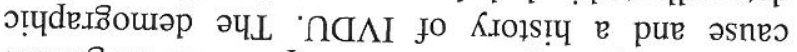

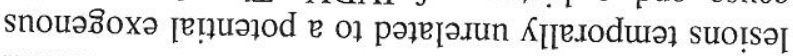

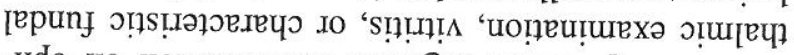

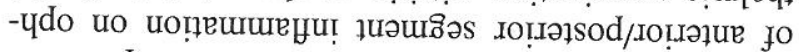

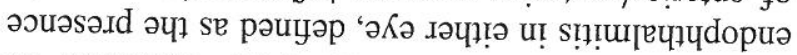

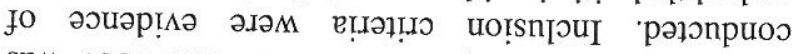

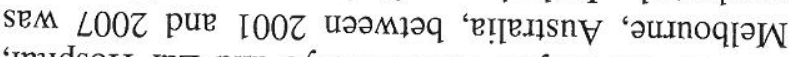
'[E]!

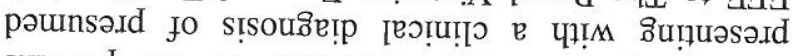

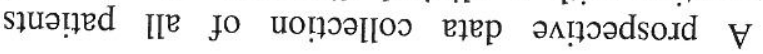

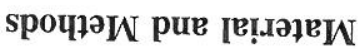

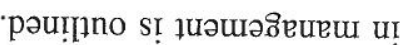

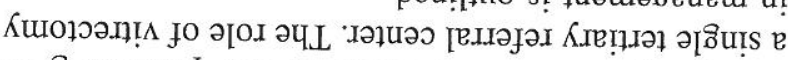

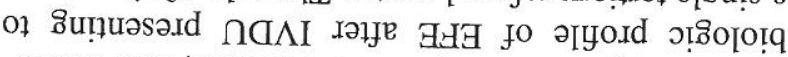

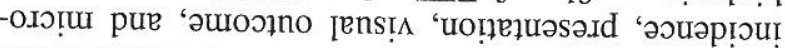

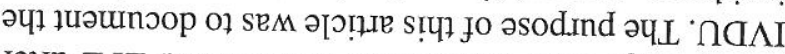

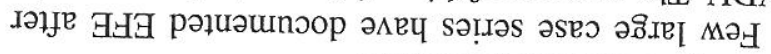

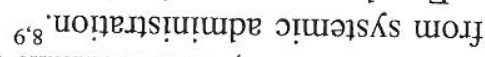

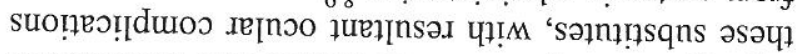

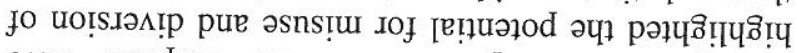

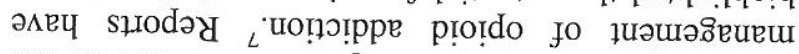

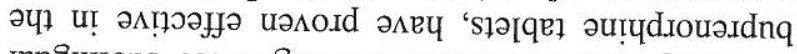

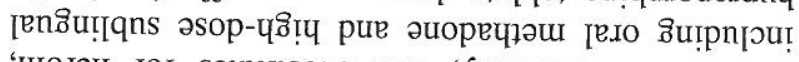

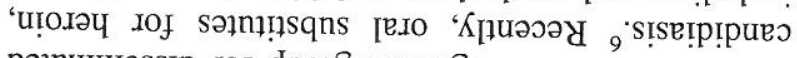

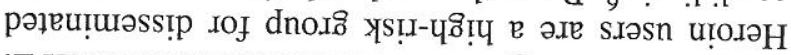

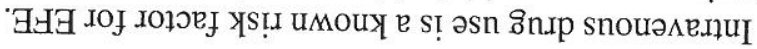


Table 1. Clinical Characteristics of Patients

\begin{tabular}{|c|c|c|c|c|c|c|c|}
\hline Case & Sex & Age & Fungus & Predisposing risk factor & Vitrectomy & Initial VA & Final VA \\
\hline 1 & $M$ & 30 & Candida albicans & IVDU & Y & $6 / 60$ & $6 / 60$ \\
\hline 2 & M & 29 & C. albicans & IVDU & $Y$ & $6 / 36$ & $6 / 18$ \\
\hline 3 & $\mathrm{~F}$ & 34 & C. albicans & IVDU & $\mathrm{N}$ & HM & $6 / 36$ \\
\hline 4 & $\mathrm{~F}$ & 31 & C. albicans & IVDU & $Y$ & $6 / 48$ & $6 / 9$ \\
\hline 5 & $\mathrm{~F}$ & 43 & C. albicans & IVDU & $\mathrm{N}$ & $2 / 60$ & $6 / 12$ \\
\hline 6 & $F$ & 29 & C. albicans & IVDU & $\mathrm{N}$ & HM & $2 / 60$ \\
\hline 7 & $\mathrm{~F}$ & 59 & C. albicans & IVDU & $\mathrm{N}$ & PL & HM \\
\hline 8 & M & 26 & Candida tropicalis & IVDU & $N$ & $\mathrm{HM}$ & $6 / 60$ \\
\hline 9 & M & 37 & C. tropicalis & IVDU & Y & $\mathrm{CF}$ & $\mathrm{HM}$ \\
\hline 10 & M & 37 & Candida dubliniensis & IVDU & Y & $6 / 6$ & $6 / 12$ \\
\hline 11 & $M$ & 33 & C. dubliniensis & IVDU & Y & $6 / 36$ & $6 / 9$ \\
\hline 12 & $M$ & 28 & C. dubliniensis & IVDU & $\mathrm{N}$ & $6 / 36$ & $6 / 6$ \\
\hline 13 & $\mathrm{~F}$ & 36 & C. dubliniensis & IVDU & $Y$ & $6 / 60$ & $\mathrm{CF}$ \\
\hline 14 & $F$ & 33 & No growth & IVDU & $N$ & $\mathrm{HM}$ & $2 / 60$ \\
\hline 15 & $M$ & 32 & No growth & IVDU & Y & HM & $2 / 60$ \\
\hline 16 & $\mathrm{~F}$ & 22 & No growth & IVDU & Y & $6 / 9$ & $6 / 6$ \\
\hline 17 & $\mathrm{~F}$ & 31 & No growth & IVDU & $\mathrm{N}$ & $6 / 6$ & $\mathrm{LtF}$ \\
\hline 18 & $M$ & 29 & No growth & IVDU & $\mathrm{N}$ & $6 / 18$ & $6 / 9$ \\
\hline 19 & $M$ & 22 & No growth & IVDU & Y & $6 / 24$ & $6 / 12$ \\
\hline
\end{tabular}

$C F$, counting fingers; F, female; HM, hand motions; LtF, lost to follow-up; M, male; N, no; PL, perception of light; $V A$, visual acuity; $Y$, yes.

IVDU was not volunteered until 48 hours post admission. The most common additional intravitreal antibiotics used were a combination of ceftazidime $(2.25 \mathrm{mg} / 0.1 \mathrm{~mL})$ and vancomycin $(1 \mathrm{mg} / \mathrm{mL})$. All patients in this series were placed on systemic oral antifungal therapy.

Final Snellen visual acuities were available for 18 of 19 patients and ranged from $6 / 6$ to hand motion with a mean visual acuity of $6 / 36$. Fifteen of 18 patients $(83.3 \%$ ) achieved a final visual acuity of $\geq 2 / 60$ (Table 1 ). In those managed with biopsy and intravitreal therapy alone, from an initial mean visual acuity of $6 / 60$, a mean final visual acuity of $6 / 36$ was recorded, with $44.5 \%$ achieving $\geq 6 / 18$. Repeat injection did not confer a worse visual outcome. In those requiring pars plana vitrectomy, from a mean presenting visual acuity of $6 / 36$, a mean final vision of $6 / 24$ was recorded with $60 \%$ achieving $\geq 6 / 18$. Overall, $50 \%$ of subjects (9 of 18) achieved a final visual acuity of $6 / 18$ or better. Culture positivity was not correlated with a worse visual prognosis.

Male patients demonstrated improved visual outcome in comparison to female subjects $(P=0.04)$. A younger age profile of $\leq 35$ years did not confer a better visual prognosis (Table 3 ).

\section{Discussion}

Endogenous fungal endophthalmitis is a rare entity, which can occur at any age and often results in severe visual loss. ${ }^{1,2,5,10}$ Bilateral involvement is often seen, particularly in those with underlying medical conditions such as immunosuppressive disorders including human immunodeficiency virus, malignancies, and associated chemotherapy and immunocompromising disease such as diabetes mellitus, renal failure, and intravenous drug abuse.

Table 2. Culture Positivity Based on Method of Sampling and Visual Results Based on Method of Treatment and Fundal View Status of 19 Patients

\begin{tabular}{|c|c|c|c|}
\hline & $\begin{array}{l}\text { Vitrectomy } \\
(n=10)\end{array}$ & $\begin{array}{l}\text { Tap/inject } \\
(\mathrm{n}=9)\end{array}$ & $P^{\star}$ \\
\hline \multicolumn{4}{|c|}{ Culture positivity (\%) } \\
\hline Culture positive & $7(70)$ & $6(66.7)$ & 0.63 \\
\hline \multirow{2}{*}{\multicolumn{4}{|c|}{$\begin{array}{l}\text { No growth } \\
\text { Visual results (1 patient lost to follow-up), \% }\end{array}$}} \\
\hline & & & 0.13 \\
\hline $\begin{array}{l}\text { Lost } \geq 1 \text { line } \\
\text { No change }\end{array}$ & $3(30)$ & & 0.13 \\
\hline $\begin{array}{l}\text { No change } \\
\text { Gain } \geq 2 \text { lines }\end{array}$ & $1(10)$ & $\begin{array}{c}0 \\
0\end{array}$ & \\
\hline \multirow{2}{*}{\multicolumn{3}{|c|}{$\begin{array}{c}\text { Gain } \geq 2 \text { lines } \\
\text { Fundal view (\%) }\end{array}$}} & \\
\hline & & $5(56)$ & 0.26 \\
\hline $\begin{array}{l}\text { Present } \\
\text { Absent }\end{array}$ & $\begin{array}{l}3(30) \\
7(70)\end{array}$ & $\begin{array}{l}5(50) \\
4(44)\end{array}$ & \\
\hline
\end{tabular}

${ }^{*} P$ from the Fisher exact test. 


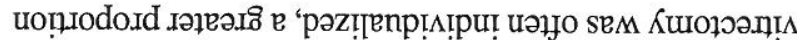

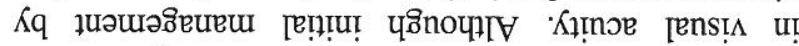

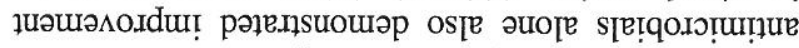
[вәп!ฺ

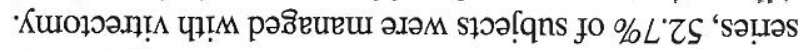

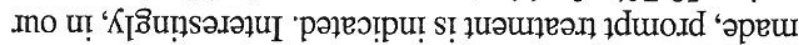

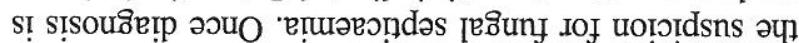

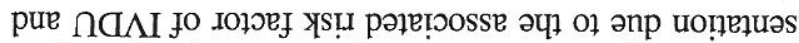

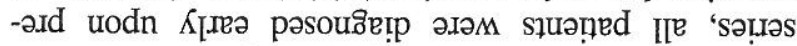

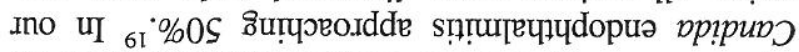

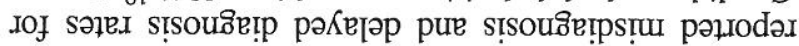

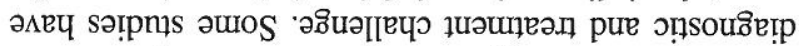

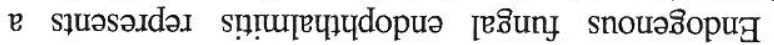

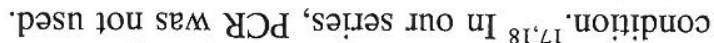

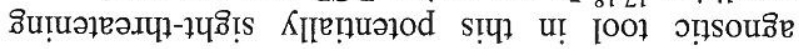

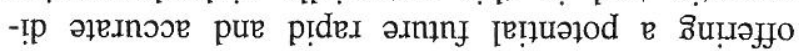

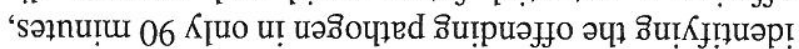

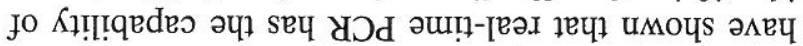

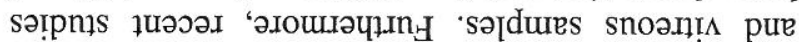

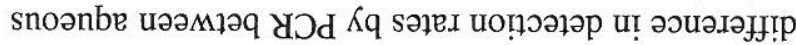

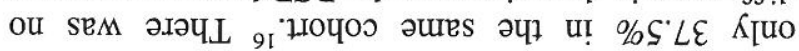

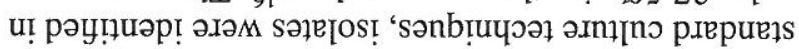

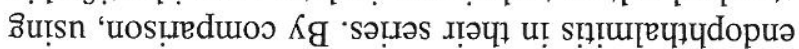

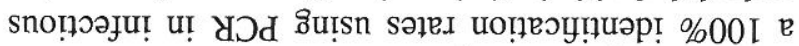

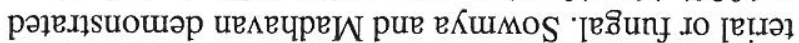

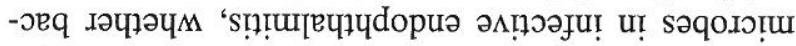

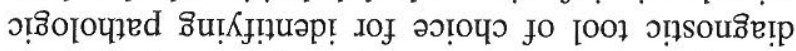

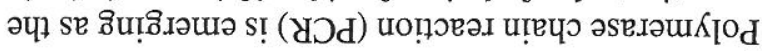

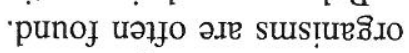

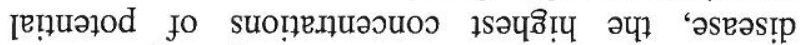

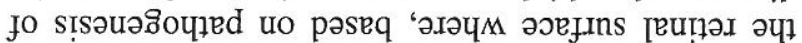

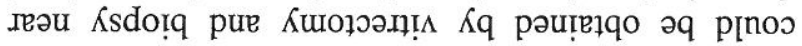

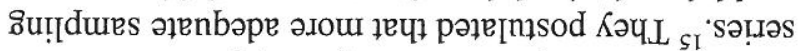

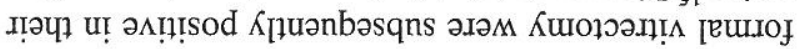

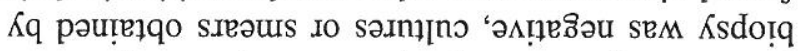

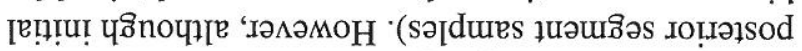

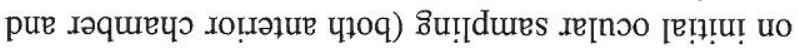

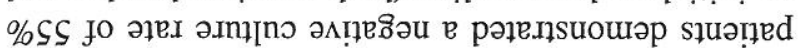

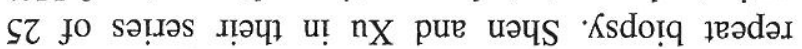

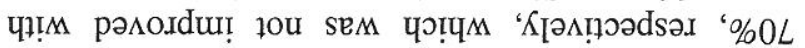

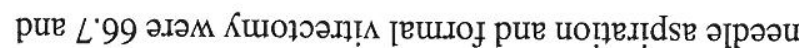

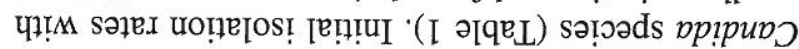

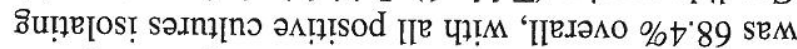

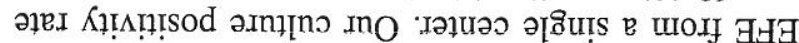

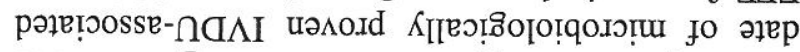

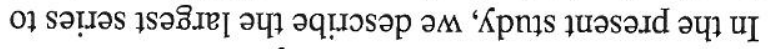

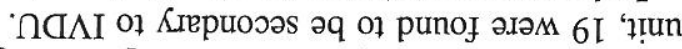

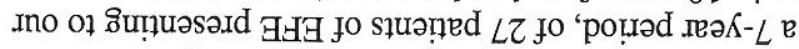

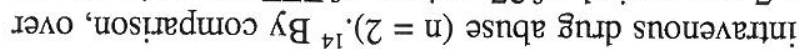

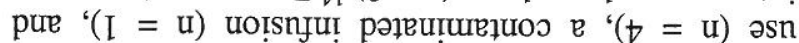

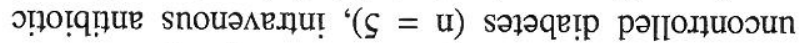

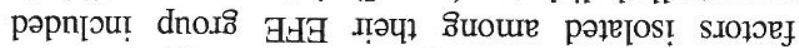

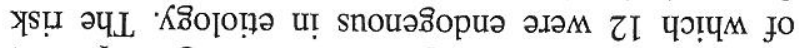

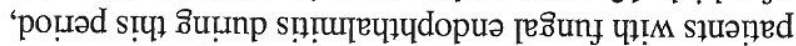

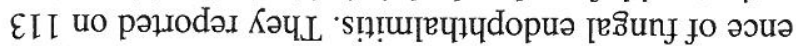

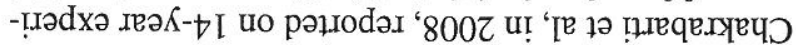

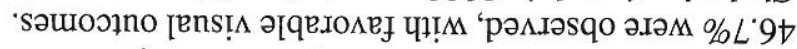

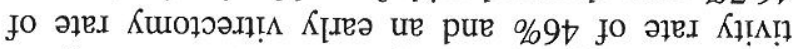

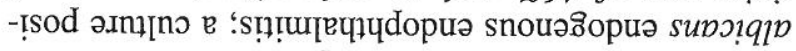

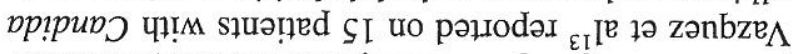

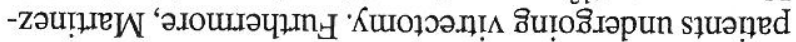

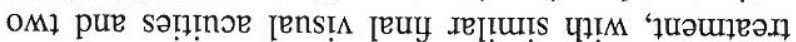

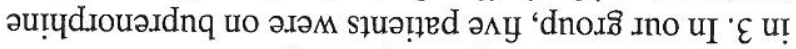

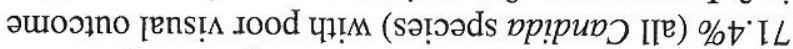

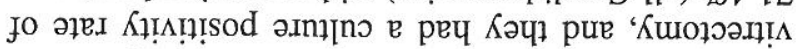

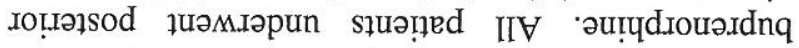

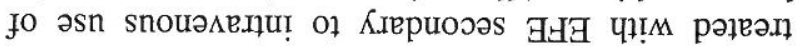

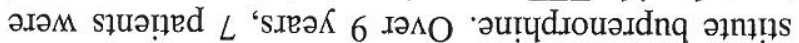

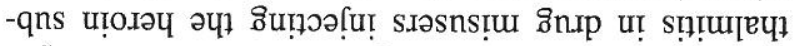

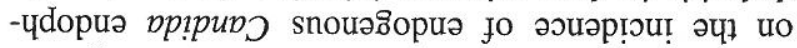

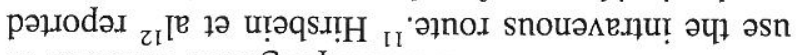

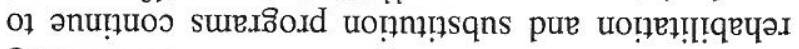

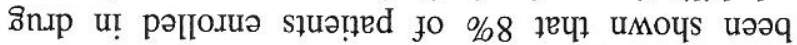

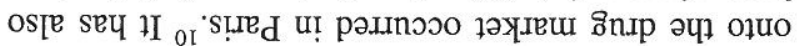

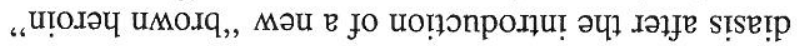

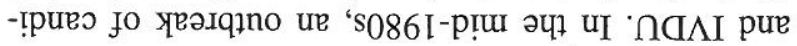

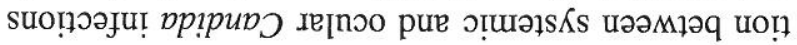

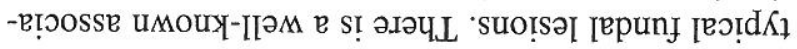
pure 8ิu!̣

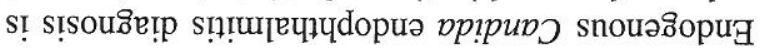

\begin{tabular}{|c|c|c|c|}
\hline & & & ${ }^{\circ} 90^{\circ} 0>d$ \\
\hline $9 t^{\circ} 0$ & (иеәш) 9ع/9 & (иеәш) 09/9 & 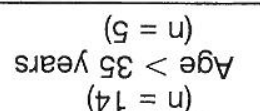 \\
\hline $6 Z^{\circ} 0$ & (uеәш) $9 \varepsilon / 9$ & (ueәш) 8t/9 & 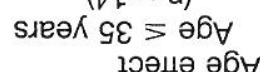 \\
\hline $\begin{array}{l}89^{\circ} 0 \\
\succ 0^{\circ} 0\end{array}$ & $\begin{array}{l}\text { (uеәш) 8t/9 } \\
\text { (ueәu) } \nabla ट / 9\end{array}$ & $\begin{array}{l}\text { (uеәш) 09/9 } \\
\text { (uеәш) 8t/9 }\end{array}$ & 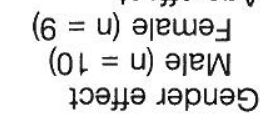 \\
\hline$d$ & $\begin{array}{c}\forall \wedge \\
\text { диәшіеәдңsod }\end{array}$ & 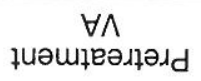 & \\
\hline
\end{tabular}

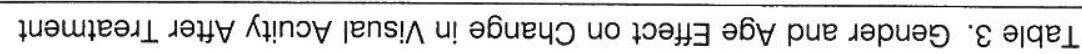


of those managed with vitrectomy presented with a poor fundal view in comparison to those managed with tap and injection alone. Tap and injection in our series was curative in 9 of 12 patients, with the remaining 3 requiring vitrectomy for lack of resolution or worsening of disease. No patients underwent enucleation. Furthermore, the association between buprenorphine and IVDU prompted a public health campaign with a subsequent lowering in incident cases (data not shown).

Visual acuity results tended to be favorable irrespective of the method of management in our series. Those patients managed with tap and injection improved over the duration of the study. In our series, $100 \%$ of patients achieved a final visual acuity greater than or equal to counting fingers, with $50 \%$ achieving $\geq 6 / 18$ visual acuity. Previous studies have reported on the benefit of vitrectomy in management. ${ }^{1,2,20-22}$ Indeed, Shen and Xu demonstrated a postsurgical visual acuity greater than or equal to counting fingers in $63 \%$ of patients managed with vitrectomy. In our series, of those treated with vitrectomy, 90\% achieved a postvitrectomy final visual acuity greater than or equal to counting fingers (despite 3 patients losing 1 line of visual acuity), with over $60 \%$ gaining $\geq 2$ lines of visual acuity and $60 \%$ achieving $\geq 6 / 18$ visual acuity. The management via vitrectomy or otherwise is difficult to elucidate in this complex condition. Indeed, few published guidelines exist on clinical criteria that would commit to a definitive management pathway in this condition. The results of the endophthalmitis vitrectomy study are not applicable directly to a disease with a very different pathogenesis. ${ }^{23,24}$ Future studies with increased numbers would be needed to address this problem.

In conclusion, IVDU-associated fungal endophthalmitis is a disease with associated ocular morbidity. A high clinical suspicion with a judicious history is necessary to allow prompt diagnosis and treatment. Visual results, in paradox to bacterial endogenous endophthalmitis, tend to be favorable.

Key words: endophthalmitis, fungal, drug abuse, microbiology, visual.

\section{References}

1. Essman TF, Flynn HW, Jr., Smiddy WE, et al. Treatment outcomes in a 10-year study of endogenous fungal endophthalmitis. Ophthalmic Surg Lasers 1997;28:185-194.

2. Feman SS, Nichols JC, Chung SM, Theobald TA. Endophthalmitis in patients with disseminated fungal disease. Trans Am Ophthalmol Soc 2002;100:67-70; discussion 70-61.

3. Okada AA, Johnson RP, Liles WC, D'Amico DJ, Baker AS. Endogenous bacterial endophthalmitis. Report of a ten-year retrospective study. Ophthalmology 1994;101:832-838.

4. Shrader SK, Band JD, Lauter CB, Murphy P. The clinical spectrum of endophthalmitis: incidence, predisposing factors, and features influencing outcome. J Infect Dis 1990;162:115-120.
5. Takebayashi K, Matsumoto S, Nakagawa Y, Wakabayashi S, Aso Y, Inukai T. Endogenous endophthalmitis and disseminated intravascular coagulation complicating a Klebsiella pneumoniae perirenal abscess in a patient with type 2 diabetes. Am J Med Sci 2005;329:157-160.

6. Mellinger M, De Beauchamp O, Gallien C, Ingold R, Taboada MJ. Epidemiological and clinical approach to the study of candidiasis caused by Candida albicans in heroin addicts in the Paris region: analysis of 35 observations. Bull Narc 1982;34: 61-81.

7. Bouchez J, Beauverie P, Touzeau D. Substitution with buprenorphine in methadone- and morphine sulfate-dependent patients. Preliminary results. Eur Addict Res 1998;1(Suppl): $8-12$.

8. Cassoux N, Bodaghi B, Lehoang P, Edel Y. Presumed ocular candidiasis in drug misusers after intravenous use of oral high dose buprenorphine (Subutex). Br J Ophthalmol 2002;86:940-941.

9. Aboltins CA, Allen P, Daffy, JR. Fungal endophthalmitis in intravenous drug users injecting buprenorphine contaminated with oral Candida species. Med J Aust 2005;182:427.

10. Dupont B, Drouhet E. Cutaneous, ocular, and osteoarticular candidiasis in heroin addicts: new clinical and therapeutic aspects in 38 patients. J Infect Dis 1985;152:577-591.

11. Leen CL, Brettle RP. Fungal infections in drug users. J Antimicrob Chemother 1991;28(Suppl A):83-96.

12. Hirsbein D, Attal P, Gueudry J, Guet I, Brasseur G, Vasseneix C. Endogenous Candida endophthalmitis in drug misusers injecting intravenous buprenorphine. J Fr Ophtalmol 2008; 31:180-183.

13. Martinez-Vazquez C, Fernandez-Ulloa J, Bordon J, et al. Candida albicans endophthalmitis in brown heroin addicts: response to early vitrectomy preceded and followed by antifungal therapy. Clin Infect Dis 1998;27:1130-1133.

14. Chakrabarti A, Shivaprakash MR, Singh R, et al. Fungal endophthalmitis: fourteen years' experience from a center in India. Retina 2008;28:1400-1407.

15. Shen $\mathrm{X}, \mathrm{Xu} \mathrm{G}$. Vitrectomy for endogenous fungal endophthalmitis. Ocul Immunol Inflamm 2009;17:148-152.

16. Sowmya P, Madhavan HN. Diagnostic utility of polymerase chain reaction on intraocular specimens to establish the etiology of infectious endophthalmitis. Eur J Ophthalmol 2009;19:812-817.

17. Goldschmidt P, Degorge S, Benallaoua D, et al. New test for the diagnosis of bacterial endophthalmitis. $\mathrm{Br} \mathrm{J}$ Ophthalmol 2009;93:1089-1095.

18. Goldschmidt P, Ferreira CC, Degorge S, et al. Rapid detection and quantification of Propionibacteriaceae. Br J Ophthalmol 2009;93:258-262.

19. Schelenz S, Goldsmith DJ. Aspergillus endophthalmitis: an unusual complication of disseminated infection in renal transplant patients. J Infect 2003;47:336-343.

20. Smiddy WE. Treatment outcomes of endogenous fungal endophthalmitis. Curr Opin Ophthalmol 1998;9:66-70.

21. Williams MA, McMullan R, Hedderwick S, Mulholland DA, Best RM. Diagnosis and treatment of endogenous fungal endophthalmitis. Ophthalmologica 2006;220:134-136.

22. Takebayashi H, Mizota A, Tanaka M. Relation between stage of endogenous fungal endophthalmitis and prognosis. Graefes Arch Clin Exp Ophthalmol 2006;244:816-820.

23. Keswani T, Ahuja V, Changulani M. Evaluation of outcome of various treatment methods for endogenous endophthalmitis. Indian J Med Sci 2006;60:454-460.

24. Verma L, Patil R, Talwar D, Tewari HK, Ravi K. First contact management of postoperative endophthalmitis. A retrospective analysis. Indian J Ophthalmol 2004;52:65-66. 